This is the accepted manuscript of the article, which has been published in Health. 2018.

https://doi.org/10.1177/1363459318785711

\title{
Expertise, advocacy and activism - a qualitative study on the activities of prostate cancer peer support workers
}

\section{Marjaana Jones, Ilkka Pietilä}

\section{Abstract}

Peer support workers are now working with patients in a variety of settings, coming into close contact and even work alongside health professionals. Despite the potentially influential position peer support workers hold in relation to those engaged in support activities, their role, duties and their relationship to peers and health professionals lack clarity and is often defined by other actors. This study explores how peer support workers interpret and define the activities, responsibilities and knowledge associated with their work. Using methods of membership categorisation analysis we analysed interview materials generated by conducting individual semi-structured interviews during the autumn of 2016 with prostate cancer peer support workers ( $\mathrm{n}=11$ ) who currently volunteer as support workers in Finland. Although the peer support workers acknowledged the psychosocial aspects of the work we argue that their interpretations extend far beyond this and encompass expertise, advocacy and activism as central aspects of their work. These can be used to strengthen their position as credible commentators and educators on issues relating to cancer and men's health; raise awareness and represent the "patient's voice" and attempt to influence both policy and clinical practice. These findings suggest that by categorising their work activities in different ways voluntary sector actors such as peer support workers can attempt portray themselves as legitimate authorities on a range of issues and influence decision making ranging from individual level treatment decisions all the way to health policy.

Key words: activism, Finland, lay expertise, patient advocacy, prostate cancer, qualitative

\section{Background}

Over the last few decades the ideas of patient centred care, patient expertise and experiential knowledge have become buzzwords in healthcare services and health policy documents in a number of Western 
countries (Coulter \& McGee, 2003; Martin, 2008). People with personal illness experiences have been able to participate in joint decision making regarding their own care (Glasdam et al., 2015) but also in the development, assessment and production of care services (Martin, 2008). In health policy rhetoric patients are often referred to as critical, responsible and empowered (Newman \& Vidler, 2006). Additionally, patient organisations and service user groups in a number of countries have been demanding to get their voices heard in healthcare and health policy decisions (Allsop, Jones \& Baggott, 2004). Lawson (2003) has highlighted a shift from the Parsonian 'sick role' that positioned the patient as a passive recipient of care, who is neither responsible for their condition nor able to participate in health related decisionmaking. In contrast to this, Lawson (20039 highlights how lived experiences are increasingly considered to offer an important contribution to the ways in which we understand health and illness. People with personal illness experiences, particularly of chronic illnesses, have even been termed experts due to the knowledge they have accumulated regarding the healthcare services, their illness, and its management (Caron-Flinterman et al., 2005). Nevertheless, it should be noted that within healthcare, the biomedical model continues to prevail with medical knowledge being regarded as general, objective and accurate in comparison to experiential or lay knowledge that is deemed subjective (Nordin, 2000).

As part of the recognition of patient illness experience, peer support has become recognised as a beneficial form of psychosocial support (Davison et al., 2000) and peer-run services have begun operating in tandem with healthcare services bringing peer support workers into clinical environments (Jacobson et al. 2012; Rebeiro Gruhl et al., 2016). Peer support has a long history and it can be linked with social movements that emphasise embodied health experiences as well as the civil/human rights movements (Mead \& MacNeil, 2006). A number of studies suggest that peer support can improve the subjective wellbeing of both people who receive it and people who provide it and strengthen participants' selfesteem, feelings of control and acceptance (Ussher et al., 2005; Gottlieb et al., 2007; Lindeman et al., 
2011). Attending peer support has been shown to produce a sense of empowerment, instill hope and confidence, facilitate coping (Docherty, 2004) and enable the sharing of experiential knowledge.

In relation to cancer, peer support can help reduce the negative or disabling effects which the illness can have on general health, relationships, coping abilities and daily functioning (Pomery et al., 2016). Previous studies focusing on peer support have described it as emotional support based on shared personal experience (Dunn et al., 2003), and non-clinical in nature (Jacobson et al., 2012). Indeed, peer support is perceived as having a non-professional vantage point (Mead \& MacNeil, 2006) and their purpose is not to provide therapy or education, despite some groups adopting aspects of psychotherapeutic and education programs (Pomery et al., 2016). From this perspective, peer support is categorised as an auxiliary activity in relation to healthcare professionals’ work and largely limited to provision of psychosocial support.

Due to the growing presence of peer support work, support activities and peer support work has been studied particularly in the field of mental health (e.g. Milligan et al., 2011; Jacobson et al., 2012; Rebeiro Gruhl et al., 2016). The work of peer support workers in relation to cancer and prostate cancer in particular, has received limited attention despite the commonality of the illness. Prostate cancer is the most common men’s cancer in Western countries (Schiff \& Mulhall, 2005) and significantly affects the lives of men and their families (Chapple \& Ziebland, 2002). Radical treatments such as surgical removal of the prostate gland and radiotherapy have common long-term side effects such as erectile, urinary and bowel dysfunctions. For many patients these side-effects remain chronic and reduce their quality of life. Since the 1990s many prostate cancer peers support groups (PCSGs) have formed to facilitate men’s adjustment and support coping with the effects of the illness (Manne, 2002). These activities are often facilitated by peer support workers, yet we know very little about the ways in which peer support workers themselves interpret their position and the duties associated with their work. 
Peer support workers are often self-selected volunteers who are motivated to help others, but who also place themselves into a new role as group leaders (Zordan et al., 2010). Within groups, peer support workers can have considerable power, responsibility and status (Smokowski et al., 2001). Prior studies have noted that peer support activities include discussions about treatment decisions and sharing of medical information such as prostate specific antigen levels (i.e. protein produced by normal and malignant cells of the prostate gland), Gleason scores and laboratory test values (Oliffe et al., 2010). Also, other men with prostate cancer are described as important providers of information and 'second opinion' (Anonymised, 2016). Although provision of medical information is not considered as part of prostate cancer peer support, in a previous study peer support workers have described medical information as a prerequisite for their work (Anonymised, 2014). These findings indicate that the work of peer support workers is not confined to sharing experiences and providing psychosocial support, which is how their work is typically described by healthcare professionals. As the working environments of peer support workers have extended outside of support groups and into hospitals, they are coming into closer contact with professional groups.

The data analysed in this study was generated through interviews conducted with peer support workers in Finland where prostate cancer peer support has established its position during the 2000s, and peer support is now offered by three cancer/patient organisations (Cancer Society of Finland, Finland's Prostate Cancer Association, Prostate Cancer Patients Support Association). Peer support can take many forms ranging from individual support to group meetings, and support via telephone, email and internet is also available, but to a lesser extent. In larger cities peer support workers are providing support also in hospitals and health centres. However, peer support does not have an established position within Finnish cancer care services and there are currently no paid positions for peer support workers, making peer support work a voluntary activity. Despite the potentially influential position the peer support workers hold, their role, duties, and their relationship to peers and health professionals lack clarity and definition. 
Hence, this study will focus on the ways in which the interview participants describe and define their work as prostate cancer peer supporter. We argue that the interpretations and definitions peer support workers offer extend beyond traditional tasks (facilitating and providing psychosocial support for peers) and encompass elements of expertise, advocacy and activism.

\section{Materials and methods}

This study is based on semi-structured individual interviews conducted with 11 men aged 52-89, who currently work as prostate cancer peer support workers. All of them had been diagnosed with and treated for prostate cancer and they had undergone peer support training organised by a patient/cancer organisations. Eight of them had been peer support workers between 5-15 years and three of them had trained as peer support workers within the last two years. The interviewees were recruited from the three organisations, which coordinate prostate cancer peer support activities in Finland and provide one to two days of training, in which all peer support workers are required to participate. The training supports support workers to run groups and covers basic principles such as confidentiality. The majority of the interviewees worked as peer support workers in larger cities within the Southern and Western parts of the country, but some provided peer support in smaller towns where they resided. All the peer support workers had been involved in individual and group support activities. Peer support workers from one organisation were also responsible for a national telephone service and peer support workers from all organisations volunteered in hospitals where they offered information and support to recently diagnosed patients. Additionally, many peer support workers gave talks and lectures at hospitals and seminars relating to prostate cancer and took part in developmental tasks such as the design of a new peer support online resource. Despite some peer support workers working in hospital settings, they remain patient/cancer organisation volunteers and are not formally part of the public health service. Prior to the interviews, ethical approval was sought from a relevant ethics committee. The interviewees were recruited by contacting the manager of each organisation who forwarded invites to current prostate 
cancer peer support workers. All participants gave verbal and written informed consent to take part in the interviews, which were completed during the autumn of 2016. All the interviews were digitally recorded, transcribed verbatim by the first author and anonymised. The interviews lasted between 44 min and 2.5 hours and they were conducted by the first author. Interview questions covered topics relating to personal motivations and experiences of both receiving and providing support (e.g. Could you tell me how you became involved in peer support work?, What support did you receive?); training; peer support activities (e.g. Could you tell me more about your work as a peer supporter? What did your training entail?) and views on prostate cancer screening. An interview guide was used flexibly to allow for free discussion on topics respondents themselves considered relevant. Both the interviews and the initial analysis process were completed in Finnish, as data analysis is more appropriate to be conducted in its original language instead of translated versions in studies which focus on how participants use language (Squires, 2009). Following the analysis, the interview extracts chosen were translated into English by the first author. Both authors speak Finnish and English fluently and the second author was able to check the accuracy of the translation. One feature of the interviews, which will be further explored in a related study, was that participants referred to the interviewer's gender (female) and emphasised the importance of being male in understanding the prostate cancer experience. As noted by Pini (2005), gender may shape an interview in several ways and the gender focus and gendered context of the research environment are critical factors in mediating the relationship between interviewer and interviewee. The peer support workers contextualised prostate cancer as an illness that deeply affects men and their masculinity.

In the analysis, we have applied methods of Membership Categorisation Analysis (MCA). We understand categories as cultural and context bound with certain activities, rights, responsibilities, knowledge and competencies attached to them (Jokinen, Juhila \& Suoninen, 2012). Chosen categories can also create moral order and status as we attach certain culturally bound assumptions to them (Sacks, 1972; Jayyusi, 1991). Silverman (2007) has argued that descriptions and categorisations should be studied from the 
perspective of the actors themselves. Hence, our emphasis is on the ways in which peer support workers describe and discuss their work. After first familiarizing ourselves with the interviews, we followed Stokoe's (2012) principles by locating explicit mentions of peer support work and descriptions attached to it. We noticed that peer support workers attached varied activities, knowledge, and competencies to their work.

In the findings section, we will outline these descriptions and highlight how peer support workers produce wide-ranging interpretations of their work in a context where they are at the same time considered as volunteers lacking a defined position or job description and still expected to engage with patients and healthcare professionals in ever expanding settings. Firstly, we will look at the expertise, which differentiates peer support workers from other men with prostate cancer and elevates them to a position resembling that of a health professional. Secondly, we will explore how peer support workers advocate for others by both educating men and representing them. Thirdly, we will focus on activities seldom discussed in relation to prostate cancer as peer support workers described their work in terms of activism and attempted to influence system level decision making and challenging existing guidelines.

\section{Findings}

As expected, the interviewees described being a peer (i.e. a fellow man who has been through prostate cancer) and a support worker (i.e. someone who has completed training and is now willing to support others with the condition) as core aspects of their work. However, in this study we wish to draw attention to the much wider plethora of activities, which peer support workers include in their work. Particularly the men who had worked as peer support workers for several years were likely to offer much wider descriptions. Men who had only recently begun working as peer support workers described their activities in traditional ways (e.g. listening, giving hope, sharing experiences) and were less likely to comment on issues they perceived as being within the realm of medicine. In this section, we will explore the activities, which peer support workers associate with their work. 


\section{Experiential knowledge is power - the expertise of peer support workers}

Throughout the interviews, peer support workers talked about possessing expertise on issues relating to prostate cancer. Peer support work is often associated with sharing of experiences, feelings and thoughts. However, the interviewees discussed the need to know more about cancer, men's health and even the healthcare system. Medical information was described as an important component of peer supporters' work and it was gathered by attending medical lectures, following media coverage, accessing online information and research databases. The interview participants were cautious of online information (chat rooms, forums) and questioned other men’s abilities to filter the relevant information from the "tsunami" of information available on the internet. As we can see in the extract below, experiential knowledge is accumulated knowledge comprising of personal experiences, other men’s experiences, and 'factual information' gathered from other sources.

Peer support worker 4: (...) each one of us seeks experiential knowledge in our own way and in my opinion that's the combination that we need. We need factual information, although we're not doctors. (...) And another thing is that we gain understanding and knowledge about the functioning of this healthcare system. How it should work and well, then we do hear and see how it's not working. But what I'm after is information (...) and real knowledge, research based knowledge, so none of these beliefs or utter nonsense but researched, clear facts.

Having expert knowledge differentiates peer support workers from other men with prostate cancer, and enables peer support workers to place themselves somewhere in between lay men (i.e. those who may have prostate cancer but not the required training, skills and knowledge to be considered experts) and healthcare professionals (who possess both training and professional status, but lack experiential knowledge). Particularly men without a cancer diagnosis and men who had a diagnosis but did not actively seek information and care were described as "passive”, "in the dark" and "not having the guts". 
In relation to this, peer support workers highlighted their own activeness and emphasised how important it was that all men would have a higher level of health literacy.

Expertise also manifested itself during peer support meetings. For example, one pair of peer support workers jokingly called their support group a "PSA academy", emphasising its educational function. This also placed an expectation on peer support workers to be able to deliver information on any issue relating to prostate cancer. The interviewees emphasised their experiential knowledge and expertise by referring to themselves as "semi-professionals" or jokingly as "mock doctors". This also meant that medical information such as laboratory results were shared at group meetings and peer support workers placed themselves into a position where they could offer opinions and advice. In the extract below, the interviewee explains that during support groups they ask new members to bring in laboratory test information and medical records. The peer support workers then go through them and make assessments on their condition. By doing so, they expand the range of activities associated with peer support by providing guidance on matters, which can be seen under realm of health professionals' work.

Peer support worker 7: When a new patient comes to the group then at first we place them in the centre and they get a chance to talk, if they are willing to talk, or we try to kind of squeeze the information out. And they get to bring up emotions and ask loads from other members. And then occasionally they don't have lots of papers [laboratory results] and we ask them, like we list the papers and ask them to bring these papers with them or to request them from the hospital. Because we can see for ourselves the figures on the paper since we've got more experience so we can because they often remember them incorrectly. And if we give information on the basis of what they remember, then we'd rather see the documentation.

The interview participants voiced criticisms about healthcare professionals' abilities and readiness to provide emotional support and sufficient information about the possible consequences of prostate cancer 
and its treatments. This allowed peer support workers to argue that their duty was to fill these gaps as they had knowledge, experience and importantly the time to spend with patients. Indeed, peer support can be seen as a way to respond to psychosocial needs of patients. However, at times the line between the functions of peer support and healthcare became blurred. One support group had begun tracking some of their members' prostate specific antigen test results, making calculations based on them and drawing their own conclusions. This was framed as an attempt to ease the doctors' workload, but it also lead to peer support workers attempting to recognise/reveal cancer prior to medical professionals.

Peer support worker 7: (...) it was one of those cases, it was stage one and we could see from the chart [follow up chart which the support worker has made] that the rate of [prostate specific antigen level] increase was really fast and we suspected it [cancer] and then he went for examinations and they found a two centimeter long renewed prostate cancer.

Although peer support workers acknowledged their lack of medical training and their position as unpaid voluntary sector workers, during the interviews they expressed criticism and suggested a number of improvements relating to treatments, professional conduct and current cancer screening practices.

Peer support worker 9: It [prostate specific antigen level testing] would cost a certain amount, but this testing system is so cheap and if and health centre staff was trained so that they would understand and take the tests and interpret them. I don't find it that difficult and these days I can interpret them much better than a doctor and I'm a lay person.

In the above extract the peer support worker refers to himself as "a lay person", yet he openly challenges current practices and presents himself as a person with sufficient skills and know-how to express these views. Nevertheless, criticising healthcare professionals was not a straightforward issue as some of the peer support workers were still undergoing treatment, which means that they were in contact with the 
doctors also as patients. Additionally, healthcare professionals volunteered to give lectures events organised by support workers and were described as valuable information resources. Potentially due to these factors, the peer support workers often directed their criticisms towards institutions, specific practices or limited resources rather than at healthcare professionals. They acknowledged the status held by professional expertise, but attempted to challenge the professional monopoly as the sole authority on prostate cancer. The extract also shows how peer support workers extended the sphere of their work outside of the traditional peer support arenas.

\section{Advocating for other men}

Building on their knowledge and skills, peer support workers also expressed that it was their task to ensure the "patient's voice" is heard. In other words, they wanted to represent and promote the interests and opinions of men with prostate cancer. Advocacy could take place in a support group setting as peer support workers wanted to ensure that men knew their rights and would be able to demand a second opinion and ask informed questions about their care.

Peer support worker 4: In a way even today when a patient goes for their meeting about the treatment decision there's not much discussion about all the possible things that can follow from this. The mode is more like three options are handed to you, chemo, operation or follow up, choose one. Depending on age a little bit, but still. You've got 15 minutes to choose and then we get started. And the patient might be pretty lost at that point really. When some of the guys somehow find their way into peer support groups at that stage when they are going about to go for the meeting. (...) Then if the guy is receptive and can handle it then we can really start to talk about our personal experience that if you choose an operation then what. Well of course technically it's the doctor who tells this. And they should tell this or otherwise I tell them to go and ask. If you feel like taking a friend with you or a wife or a son or anyone or one of us peers. 
Here the work of a peer support worker is described as instrumental in ensuring men get sufficient information. It is even suggested that instead of doctors, it is the peer support workers who provide truly patient centred care and support as they have time to discuss treatment options. In the extract, the interviewee also notes how the provision of this information belongs within the medical remit. In order to justify stepping over this boundary he refers to the lack of time and resources professionals have which justifies peer support workers to adopt responsibilities which “technically” belong to the doctor. Peer support workers also discussed how they are able to support and advocate for men by attending hospital appointments jointly with them. A central aim was to create informed and critical patients who ask appropriate questions and deal with professionals and the healthcare system in a manner, which provides the best outcome. Peer support workers encourage men to seek a second opinion, particularly from doctors working in the private sector. The second opinion or statement could then be used to challenge the views of the doctor in charge of their treatment.

Peer support worker 10: We've got this unwritten agreement that at least from [names a private doctor] if you go and ask you can get like instructions or a statement. Then when you get the statement you take it to hospital and when the urologist starts to probe you, then you give them the statement and say that [private doctor] has done this and then they change their stance. But not everyone has the courage, it takes guts and some are afraid that they're gonna get mad and leave them in a bad way. And what we are trying to do in peer support is to guide them to keep their own mind. Don't be rude but say things in a smart manner and stick to the facts.

In addition to these tasks, advocacy work placed peer support workers into new work settings. As most health professionals lacked a personal experience of cancer, peer support workers were attempting to increase professionals' awareness of the issues that affect men with prostate cancer. Some of the 
interviewees attended conferences and other events where they gave lectures to professionals. As one peer support worker discusses his talk at a medical conference:

Peer support worker 6: (...) last time in Oulu [a city in northern Finland] there was this one really tough urologist who thought that patients don't know anything. So then he came to me afterwards and said that this information is really valuable, because they can't be sitting with the patient afterwards and asking how they feel.

A level of authority is still attached to the category of a doctor or healthcare professional and hence recognition from them could be used to strengthen the legitimacy of peer support workers and experiential knowledge. In the interview accounts, peer support workers expressed concerns about the general attitudes they believed men had towards health. These concerns did not solely relate to the men they met through peer support, but to men in general as all of them could be regarded as potential future patients. In contrast, peer support workers described themselves as active and capable of seeking support and care, but suggested that most men did not act in this way and hence for various reasons men could miss out on receiving timely treatment. Hence, peer support workers facilitated informal opportunities for men to meet with doctors and ask questions following public events such as lectures. As mentioned earlier, advocacy could also take place outside the arenas of healthcare and voluntary sector activities. Most of the interviewees had participated in TV, magazine and newspaper interviews as they were attempting to raise general awareness of prostate cancer and decrease the potential shame and stigma associated with the illness.

Peer support worker 5: Well, the aim of the photo exhibition was to give prostate cancer a face so it was like a really concrete thing. This illness is not like other things so it needs a face because so many men are ashamed of it. So I'm ready to give a face to the thing... Giving a face to cancer also meant that the peer support workers could become recognizable in their local communities. Many interviewees described incidences where colleagues, family members, friends and 
acquaintances had approached them following public appearances. This extended the scope of peer supporters' work as they were providing support, guidance and information during their spare time, outside of their official peer support duties. Awareness raising through public appearances and by lending one's face (and body) to the cause also connects with patient activism and many similarities can be drawn to the activities of the breast cancer movement (King, 2004), which has not only raised awareness but also engaged politically with issues relating to cancer.

\section{Fighting for access - the activism of peer support workers}

Expertise could produce credibility and status, whereas advocacy focused on awareness raising and conveying the issues affecting men to the professionals treating them. Activism moves peer support workers even further away from support group settings and into national and international arenas. Furthermore, these activities are far removed from providing support and are aimed at influencing services on a national level with regards to treatment, medication accessibility and best practice guidelines. Peer support workers were prepared to challenge existing guidelines and practices, including organising prostate specific antigen screening. Patient organisations do not presume that peer support workers participate in these activities. Nevertheless, the interviewees themselves described it as their “duty" or a "mission” to make a difference on a wider scale. Over the past decade, there have been increasingly more opportunities for patients to take part in healthcare service development work. Peer support workers viewed this as an opportunity to bring the voice of experience to the fore and were actively engaging in assessment and development work.

Peer support worker 4: (...) The point isn't to tell that the health centre doctor is bad and doing things wrong and this should be like that. It's about bringing information and being active. So I believe that bit by bit this develops the system further. And we peers and patients in my opinion are in a valuable position. We've got the chance and no one else can do it. 
In the extract, the interviewee emphasises the uniqueness of experience and emphasises people with personal illness experience as a force for change. Placing experiential knowledge and patient views at the centre of service development is described as the only way forwards and decision makers' and health professionals’ abilities to promote the best interest of patients is questioned.

Peer support worker 11: So for example medicine is such a commotion and politicians give their own flavour to the mix. As we fight which medications are available and what we need to pay for them. So we've got lots of topics of interest. I don't know if these are even peer support issues these things but they're important nevertheless.

Medication availability could be considered outside of the remit of peer support work. Nevertheless, the interviewees included it within their tasks and expressed their commitment in strong terms, i.e. "we fight" to gain access to medication. Peer support workers also aimed to present themselves as credible participants in decision making by referencing places such as the Finnish parliament where one group of peer support workers had attended to express their views on medication costs and availability.

Peer support worker 7: But one important thing in this medication side which we didn't talk about is that here in Finland we have a problematic situation in the public sector where you need to follow certain steps during treatment (...) But we've been to parliament to talk to these and had meetings with them how to adjust this. There are a few medications, one of them is Enzalutamide and the other is Abiraterone.

Other peer support workers had widened their work beyond the national borders, as one interviewee discusses attending the European Parliament as a patient representative, an activist with networks covering numerous countries and as an expert who is able to moderate discussions on prostate cancer.

Peer support worker 6: From the very beginning I've been one of the 15 founders of, I'm praising myself a bit here, this [European organisation], which is now in 23 or $27 \mathrm{EU}$ 
countries. (...) And last year we were at the European Parliament and I got to be a moderator in one of our things where we discussed prostate cancer.

Peer support workers were also promoting issues, which may not have direct influence on them. Many of them no longer required medication or access to latest treatments. However, they wanted to make changes, which could affect all men with or without current prostate cancer diagnosis. One way of doing this was trying to make changes to the Best Practice Guidelines.

Peer support worker 7: And the best practice guideline is unfinished and its emphasis is on urology and on the early stages of illness. Then this latter stage, well we've [group of support workers] discussed it and we've even got a small team here, so that we could get these clear [prostate specific antigen] ranges included in the guideline...

This continues to highlight how some peer support workers appeared comfortable commenting on medical issues and even attempting to make changes to practice guidelines. By doing so, the interviewees also attempted to redefine the remit of their knowledge and activities associated with peer support work. Other forms of activism included fundraising activities. In the treatment of prostate cancer, surgical robots have only been in use in Finnish hospitals since the late 2000s. Patient organisations, and peer support workers as the active members of these organisations, were strongly in favour of robot purchases, campaigning and raising money for the cause.

Peer support worker 9: By the way the patient organisation drove through this robot thing, this is a slightly different issues, but the organisation began to push through this issue of purchasing a robot when I came, when I stepped into the peer support serv-came to peer support meetings. It was a huge venture and a difficult venture as doctors [within the hospital district] from managers to senior physicians were against it. And urologists opposed it as well. But then urologists are just surgeons and it's understandable that they'll never learn to do robot surgeries. (...) Well, the robot, we got the robot. The board 
of the hospital district made the decision that the robots would be purchased and then these turncoats began to appear slowly. But the patient organisation was forgotten in that respect that the organisation was the father of the whole idea and even raised a substantial sum of money for it.

In this extract, patient organisations and their active members are positioned as forerunners, whereas healthcare management and medical practitioners are suggested to be holding back progress due to their own careerist concerns. The extract also highlights another issue reflected in several interview accounts. Despite their active role, both patient organisations and their active members could be moved aside and their contributions ignored due to their un-established position. Although the majority of the activities relating to patient activism were conducted in collaboration with healthcare professionals, healthcare management, policy makers and members of other patient organisations, peer support workers discussed their willingness to engage in activities which openly challenge the current guidelines and practices. One example of this relates to population based prostate specific antigen screening, which is not recommended in Finnish guidelines.

Peer support worker9: Definitely screening. I've been supporting that all along. I've just been waiting for a suitable time that the organisation could campaign for screening again. And it's close. Once we get this health and social care restructuring mess out of the way then we could do this screening. (...) It's not as expensive as they think. And the decisions which was made before was done in the previous decade and it's a decision which reflects that point in time and it was incorrect to begin with.

Open support for screening was expressed by almost all of the interviewees, although a few of them expressed reservations. The peer support workers also encouraged men to get tested regularly and one organisation had for a number of years been organising regular testing sessions which were open to the public. On issues relating to care and access to latest treatments, peer support workers were likely to 
present themselves as forerunners who were actively pushing changes, which could benefit current and future patients. When required they were also prepared to confront opposition and challenge existing practices.

\section{Discussion}

In this study, we have explored the ways in which prostate cancer peer support workers describe and define their work. We wanted to particularly highlight how interview participants expanded the range of competencies, responsibilities, and knowledge associated with peer support work. Katz and Bender (1976) have suggested that support groups can be classified as 'inner-focused' or 'outer-focused' depending on whether their main concern is providing members with an opportunity to share personal problems and feelings or whether they concentrate on acting as a pressure group. This can offer one way of understanding the work of peer supporters, who in this study often identified both of these focuses as central aspects of their work activities. With peers, they wanted to promote sharing and support, however, in most cases peer support workers described it as their duty to also educate men, communicate new information to them and make their voices heard in healthcare and on a policy level.

Ziebland et al. (2004) have discussed the emergence of a felt imperative to be, or present oneself as, an expert and a critical patient who is able to question advice and locate effective treatments. Peer support workers talked about possessing experience based knowledge, which provided them with expertise. This included explicit knowledge comprising of facts and propositions, such as medical information about cancer and combines it with tactic knowledge comprising of ideas and experiences, such as personal experience of illness and how to navigate healthcare services (Foster, 2016). Including expertise as an important component of peer support work is not a surprising finding as patient expertise and the image of an active, self-sufficient and knowledgeable healthcare user are promoted widely through health policies in many countries, including Finland (Coulter \& McGee, 2003; Newman \& Vidler, 2006; Leppo \& Perälä, 2009). Expertise played an important role in defining peer support workers’ relationship to 
other men and to health professionals. Other men, who had not gathered the same amount of experiences and information, were portrayed as less informed and in need of advice, information and guidance on social, emotional, practical and at times even medical issues. This also led to situations where peer support workers positioned themselves as authoritative figures, who could make comments on issues usually regarded as under the realm of health professionals. For example, some of the interviewees described the collecting and sharing of laboratory results as part of support group activities. Expertise based on lived experiences and accumulated knowledge was also emphasised in relation to health professionals. Prior (2003) has suggested that in order to challenge medical hegemony, lay people are required to turn themselves into experts. In order to legitimise their own position, peer support workers also questioned whether healthcare professionals have the correct resources, skills or experiences to address social and emotional aspects of the illness and to promote patient’s interests.

Hagan and Donovan (2013) have argued that within the context of cancer, survivors are often engaging in self-advocacy as they are required to navigate obstacles associated with different stages of survivorship, including fragmented healthcare systems and array of information. In this study, we have argued that peer support workers build on this self-advocacy and adopt a position where they can advocate not only for themselves, but also for other men. Patient advocacy is not a new issue and prior studies have discussed advocacy as part of peer support activities (Jacobson, 2012) but in the context of prostate cancer this aspect has received limited attention. Oliffe et al. (2010) have noted the consumerist elements of prostate cancer peer support and described men engaging in activities such as ‘shopping around' for best doctors, purchasing alternative medication or contesting hierarchical doctor-patient interactions. Although similar activities were also present in our study, it might be an oversimplification to describe the advocacy work of peer support workers merely in terms of consumerism. Giving hope, increasing awareness, strengthening men's health literacy and empowering others with prostate cancer were described as strong motivating factors for support work. Advocacy could also mean peer support workers creating informal 
spaces where men could interact with medical professionals and attempting to bring patients' views to the attention of the professionals treating them. Additionally, advocacy also meant that peer support workers actively sought to improve the care and treatment men received. They were willing to speak on behalf of others and wanted their input to be valued. As one interviewee expressed it:"this kind of patient advocate is not much worse than a doctor, we've both got a lot to say”. Attaching expertise and advocacy to the work of peer supporters also brought them closer to a semi-professional position. Their descriptions placed them somewhere in between established health professionals and lay people as they had vast amounts of experiential, factual and tactical knowledge but lacked a formal training (apart from their short peer support course) and a formal position within healthcare.

Our findings also highlight peer support workers’ promotion of patient involvement and deployment of experiential knowledge in service development. Coupled with advocacy and educational activities associated with the work, these findings lead us to align prostate cancer peer supporters with other social movements in health, including HIV/AIDS and breast cancer activism (see e.g. Epstein, 1995; King, 2004; Milligan et al., 2011). The interviewees joined in with the arguments that experiences should be considered as legitimate sources of knowledge with respect to definitions of illness and measurement of the efficacy and value of treatments. In prior studies, evidence has been presented for and against the potential of peer support to challenge medical dominance and present a different perspective (e.g. Yaskowich \& Stam, 2003; Kelleher, 2006). As mentioned earlier in this article, peer support groups offer people opportunities to discuss their experiences, stigma, and worries about incorporating the illness into their social identity. As Kelleher (2004) has argued, this poses criticism on medicine’s narrow focus on the experiences of trying to live and work with the condition.

Additionally, peer support workers raised funds, campaigned for a number of issues, and attempted to influence national level decision making on matters such as medication availability and best practice guidelines. They also challenged ideas regarding men's health behavior (i.e. men do not seek help or are 
not interested in health) by engaging in activities that were aimed at educating men and by claiming that it was men's responsibility to be aware of their health and possible illnesses they may have. Although support work activities can be interpreted along these lines, it should be noted that they also wanted to collaborate with health professionals and doctors in particular. Indeed, many of the organisations peer supporters worked for has health professionals in their boards. In addition, peer support workers presented arguments for increased screening and wanted quicker access to new medications and treatments. It is difficult to interpret these actions as a challenge to medicine as such. Arguing for screening does go against the current national guidelines, yet it can be seen as a way to increase medical surveillance measures. Hence, although the actions peer supporters associated with their work had many similarities with the actions of other activist groups or social movements, they were also connected to health professionals and could even promote increased surveillance in the form of prostate specific antigen testing or closer personal monitoring of laboratory results.

This has been a small scale study focusing on the activities of prostate cancer peer support workers. Although the age range, geographical location and time they had worked as peer supporters varied, the number of interview participants sets limitations to the study. However, the number of participants also reflects the current situation in prostate cancer peer support as patient organisations experience difficulties in recruiting more support workers. The men who chose to participate may be the ones who are most actively engaged and vocal about their work. Hence, the views of peer support workers who may be less outspoken, not keen to participate in studies or perhaps content with running their groups are not presented. During the analysis, it was also noted that interview participants who had been peer support workers for a longer period were particularly likely to offer wider interpretations of their work. This could indicate that the way in which peer support work is interpreted varies depending on how long one has worked as a peer supporter. However, we acknowledge that due to the limitations of interview data we are 
unable to make any generalisations regarding this matter, but it does raise new questions about the development of the peer support worker role over time.

\section{Conclusions}

In this study, we have explored ways in which peer support workers describe the activities, responsibilities, and knowledge associated with their work. We focused particularly on the aspects, which moved the work outside of the traditional tasks of listening and providing psychosocial support. Ideas of patient expertise and experiential knowledge were strongly reflected in the interview accounts and expertise brings with it a new level of credibility. Expertise was strongly intertwined with advocating for other men and attempting to make changes to healthcare services and even health policy. Peer support workers networked with health professionals but also wanted to challenge current healthcare practices, whilst encouraging men to become critical, knowledgeable and empowered. In addition, they attempted to challenge health professionals as the only source of knowledge on matters related to prostate cancer and men’s wellbeing and called for a more holistic approach to patient care.

\section{Funding}

The authors received no financial support for the research, authorship, and/or publication of this article.

\section{References}

Allsop J, Jones K and Baggott R (2004) Health consumer groups in the UK: a new social movement? Sociology of Health and Illness 26(6): 737-756.

Anonymised (2014) Details omitted for double-blind reviewing. Anonymised (2016) Details omitted for double-blind reviewing.

Caron-Flinterman JF, Broerse JWE and Bunders JFG (2005) The experiential knowledge of patients: a new resource for biomedical research? Social Science \& Medicine 60: 2575-2584.

Chapple A and Ziebland S (2002) Prostate cancer: embodied experiences and perceptions of masculinity. Sociology of Health and Illness 24(6): 820-841. 
Coulter A and Magee H (2003) The European Patient of the Future. Picker Institute Europe. Maidenhead: Open University Press.

Davison K P, Pennebaker JW and Dickerson SS (2000) Who talks? The social psychology of illness support groups. American Psychologist 55(2): 205-217.

Docherty A (2004) Experience, functions and benefits of a cancer support group. Patient Education \& Counseling 55(1): 87-93.

Dunn J, Steginga SK, Rosoman N and Millichap D (2003) A Review of Peer Support in the Context of Cancer. Journal of Psychosocial Oncology 21(2): 55-67.

Epstein S (1995) The Construction of Lay Expertise: AIDS Activism and the Forging of Credibility in the Reform of Clinical Trials. Science, Technology, \& Human Values 20(4): 408-437.

Foster, D. (2016) ‘Keep complaining til someone listens’: Exchanges of tacit healthcare knowledge in online illness communities. Social Science \& Medicine 166: $25 \mathrm{e} 32$.

Glasdam S, Oeye C and Thrysoee L (2015) Patients’ participation in decision-making in the medical field - 'projectification' of patients in a neoliberal framed healthcare system. Nursing Philosophy 16: 226-238. Gottlieb B and Wachala ED (2007) Cancer support groups: a critical review of empirical studies. PsychoOncology 16: 379-400.

Hagan TL and Donovan HS (2013) Self-advocacy and cancer: a concept analysis. Journal of Advanced Nursing 69(10): 2348-59.

Jacobson N, Trojanowski L and Dewa CS (2012) What do peer support workers do? A job description. BMC Health Services Research 12: 205.

Jayyusi L (1991) Values and moral judgement: communicative praxis as moral order. In G Button (ed.) Ethnomethodology and the Human Sciences. Cambridge: Cambridge University Press. pp. 227-251.

Katz A and Bender E (1976) The strength in us. New York: New Viewpoints. 
Kelleher D (2006) Self-help groups and their relationship to medicine. In D Kelleher, J Gabe and G Williams (eds.) Challenging Medicine (2nd ed.). London \& New York: Routledge. pp. 104-121.

King S (2004) Pink Ribbons Inc: breast cancer activism and the politics of philanthropy. International Journal of Qualitative Studies in Education 17(4): 473-492.

Lawton J (2003) Lay experiences of health and illness: past research and future agendas. Sociology of Health and Illness 25: 23-40.

Leppo A and Perälä R (2009) User involvement in Finland: the hybrid of control and emancipation. Journal of Health Organization and Management 23(3): 359-371.

Lindeman C, Strang P and Lekander M (2011) Support group for cancer patients. Does it improve their physical and psychological wellbeing? A pilot study. Support Care Cancer 113: 652-7.

Manne SL (2002) Prostate cancer support and advocacy groups: their role for patients and family members. Seminars of Urologic Oncology 20(1): 45-54.

Martin GP (2008) ‘Ordinary people only’: knowledge, representativeness, and the publics of public participation in healthcare. Sociology of Health and Illness 30(1): 35-54.

Mead S and MacNeil C (2006) Peer support: what makes it unique. International Journal of Psychosocial Rehabilitation 10: 29-37.

Milligan C, Kearns R and Kyle RG (2011) Unpacking stored and storied knowledge: Elicited biographies of activism in mental health. Health \& Place 17(1): 7-16.

Newman J and Vidler E (2006) Discriminating Customers, Responsible Patients, Empowered Users: Consumerism and the Modernisation of Health Care. Journal of Social Policy 35(2): 193-209. Nordin I (2000) Expert and non-expert knowledge in medical practice. Medicine, Health Care and Philosophy 3: 297-304. 
Oliffe JL, Bottorff JL, McKenzie MM, Hislop TG, Gerbrandt JS and Oglov V (2010) Prostate cancer support groups, health literacy and consumerism: Are community-based volunteers re-defining older men's health? Health 15(6):555-570.

Pini B (2005) Interviewing men. Gender and the collection and interpretation of qualitative data. Journal of Sociology 41(2): 201-216.

Pomery A, Schofield P, Xhilaga M and Gough K (2016) Skills, knowledge and attributes of support group leaders: A systematic review. Patient Education and Counseling 99(5): 672-688.

Rebeiro Gruhl KL, LaCarte S and Calixte S (2016) Authentic peer support work: challenges and opportunities for an evolving occupation. Journal of Mental Health 25(1): 78-86.

Sacks H (1972) On the analyzability of stories by children. In J Coulter (ed.) Ethnomethodological Sociology. Aldershot: Edward Elgar Publishing Company. pp. 254-270.

Schiff JD and Mulhall JP (2005) Neuroprotective Strategies in Radical Prostatectomy. British Journal of Urology International 95(1): 11-14.

Silverman D (2007) A very short, fairly interesting and reasonably cheap book about qualitative research. London: Sage Publications.

Smokowski P, Rose SD and Baccallao ML (2001) Damaging experiences in therapeutic groups: how vulnerable consumers become group casualties. Small Group Research 20: 205-217.

Squires A (2009) Methodological challenges in cross-cultural qualitative research: a research review. International Journal of Nursing Studies 46: 227-287.

Stokoe E (2012) Moving forward with membership categorization analysis: Methods for systematic analysis. Discourse Studies 14(3): 227-303.

Ussher J, Kirsten L, Butow P and Sandoval M (2005) What do cancer support groups provide which other supportive relationships do not? The experience of peer support groups for people with cancer. Social Science and Medicine 62: 2565-2576. 
Yaskowich KM and Stam HJ (2003) Cancer narratives and the cancer support group. Journal of Health Psychology 8(6): 720-37.

Ziebland S, Chapple A, Dumelow C, Evans J, Prinjha S and Rozmovits L (2004) How the internet affects patients’ experiences of cancer: a qualitative study. BMJ 328(7439): 564.

Zordan RD, Juraskova I, Butow PN, Jolan A, Kirsten L, Chapman J et al. (2010) Exploring the impacts of training on the experience of Australian support group leaders: current practices and implications for research. Health Expectations 13: 427-440. 